\title{
Alcune osservazioni a seguito di una nuova edizione del Viaggio della Serenissima S. Bona Regina in Polonia di C. Carmignano*
}

\section{Abstract \\ Some Reflections on a New Edition of C. Carmignano's Viaggio della Serenissima S. Bona Regina in Polonia}

The article is a review to Andrea Colelli (ed.), C. Carmignano: Viaggio della Serenissima S. Bona Regina in Polonia, con nota introduttiva di Luigi Marinelli. Roma, 2018. In discussing the edition, proposals are put forward for what the intertextual dimension of the poem is concerned, drawing attention on the similarities between the first 'capitulo' of the poem and Joannes Secundus' elegy I 8, both texts being denied epithalamia.

Key words: Carmignano, Bona Sforza, Andrea Colelli, Luigi Marinelli, Polish Literature, Italian Literature

Parole chiave: Carmignano, Bona Sforza, Andrea Colelli, Luigi Marinelli, letteratura polacca, letteratura italiana

\footnotetext{
* Colantonio Carmignano: Viaggio della Serenissima S. Bona Regina in Polonia. A cura di A. Colelli,
} con una nota introduttiva di Luigi Marinelli. Roma 2018, 158 p.

185

Fabrica Litterarum Polono-Italica | 2020, nr 1 (2)

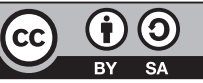


Bona Sforza duchessa di Bari, figlia di Gian Galeazzo Sforza e Isabella d'Aragona nacque a Napoli nel 1494; sempre a Napoli, ventitreenne, il 6 dicembre 1517, aveva sposato per procura a Castel Capuano Sigismondo I Jagellone (1467-1548) e il 3 febbraio 1518 aveva preso la via del mare salpando da Manfredonia, onde poi raggiungere, dopo aver navigato per un paio di settimane, il porto di Fiume e proseguire il viaggio verso la Polonia via terra. A Cracovia Bona giungerà il 15 aprile 1518, dopo aver attraversato i territori dell'odierna Slovenia, dell'Austria, della Boemia. II matrimonio ufficiale verrà celebrato tre giorni dopo, il 18 aprile, e Bona regnerà accanto al marito fino alla morte del consorte, durante gli anni di vedovanza intrattenendo rapporti tesissimi con il figlio, Sigismondo II, che le rinfacciava di essersi opposta alle di lui nozze con Barbara Radziwiłł. Fuggita allora alla volta di Bari (1556) portando con sé 430.000 ducati per affidarli a Carlo V nella speranza d'essere nominata Viceré di Napoli, Bona morì sola nel suo sfarzoso castello di Bari, così come descritto in un frammento dello storico Klemens Kantecki riportato da Luigi Marinelli nella sua introduzione:

Giaceva su un letto sontuoso pieno di addobbi, ma intorno a lei non v'era traccia di quella premura di cui beneficiano persino i più miseri nel momento in cui si approssima la loro fine; anzi, stava agonizzando fra la più triste desolazione e abbandono. Nessuno piangeva la moribonda, nessuno cercava di alleviarle le sofferenze dell'ultima ora (Colelli-Marinelli 2018: 9).

Una misera fine ma che al contempo è anche una fine degna di un'eroina tragica e del resto la protagonista della nostra storia finì avvelenata probabilmente da Gian Lorenzo Pappacoda che tentò anche di farle firmare, ormai in punto di morte, un testamento a favore degli Asburgo. Si spense il 19 novembre 1557.

Un po' come tutte le eroine tragiche, Bona era un personaggio ricco di chiaroscuri: responsabile principale dell'afflusso massiccio di intellettuali e di esponenti della cultura italiana alla corte di Cracovia, e per questo da un lato ammirata, era detestata dall'altro in quanto donna e straniera, nonché in quanto personalità politicamente attiva a sostegno del rafforzamento dei poteri monarchici, come riportato da Marinelli sulla scorta di Tazbir (Colelli-Marinelli 2018: 16).

Il libro che qui si recensisce è un'edizione del prosimetro Viaggio della Serenissima S. Donna Bona Regina [...] in Polonia, vergato da Colantonio Carmignano (che prese poi a firmare le sue opere con lo pseudonimo di Parthenopeus Suavius) su insistenza di Prospero Colonna, capitano di ventura che segui Bona Sforza in Polonia e che si diede pena affinché il viaggio sortisse buon esito.

Per quanto riguarda il Carmignano, poche sono le informazioni biografiche di cui disponiamo: di nobile famiglia napoletana, il padre Andrea aveva avuto rapporti con la madre di Bona Sforza, Isabella d'Aragona e non ci sono dubbi che il 
poeta fosse ammiratore di Sannazaro. Sappiamo poi Carmignano tesoriere di Bona Sforza nel 1518 e al suo seguito durante il viaggio in Polonia. La morte lo colse probabilmente nel 1544.

Per quanto concerne il poemetto che Colelli ha pubblicato e commentato invece, è uscito a stampa a Bari nel 1535 nel volume Operette del Parthenopeo Suavio in vari tempi et per diversi subietti composte, et de Silvan Flammineo insiemi raccolte, et alla amorosa et moral sua Calamita intitulate, il quale risulta peraltro essere il primo libro stampato in Puglia di cui si abbia notizia certa (Colelli-Marinelli 2018: 134). II testo, a cui erano già toccati gli onori di un'edizione moderna (seppur parziale) ad opera di Isabella Nuovo (Nuovo 2007), descrive il viaggio di Bona dalla Puglia alla Polonia e si compone d'una dedica in prosa, d'un sonetto indirizzati ad Isabella d'Aragona (nella dedica Carmignano non perde occasione di ringraziare anche Prospero Colonna), nonché di quindici "capituli" in terza rima, in cui viene raccontato il viaggio di Bona e del suo seguito.

Carmignano è senz'altro un poeta minore del Cinquecento italiano, ciò di cui s'era reso conto già Dionisotti riflettendo sulla prima edizione veneziana del 1516 dei lavori del poeta, uscita con il titolo Le cose vulgare e che lo studioso finì per stroncare senza troppi complimenti (Colelli-Marinelli 2018: 11). Tuttavia Dionisotti ha forse esagerato in severità, ché in quel volume del 1516 mancava ad esempio proprio il poemetto di cui ora si discute e che sì, in alcuni tratti risulta procedere faticosamente, ma è capace di momenti di apprezzabile verve poetica, come del resto ben messo in luce da Colelli e Marinelli nei loro rispettivi saggi, nonché rilevato dal primo dei due nelle note di commento. Mi limiterò qui a offrire qualche spunto, già notato da Colelli, e ad aggiungere alcune mie impressioni di lettura, senza la pretesa di rintracciare tracce intertestuali o suggestioni imitative cogenti dietro ai versi del Carmignano.

Anzitutto, è bene ricordare le giuste "consonanze nel segno di Dante", mi si passi l'espressione, evidenziate da Colelli: così come il pellegrino della Commedia inizia il suo viaggio da una selva oscura per poi ascendere in Paradiso a vedere "I'amor che move il sole e l'altre stelle", anche Bona Sforza parte sotto pessimi auspici (il mare in tempesta) e giunge a destinazione con il favore degli dei (ci tornerò subito) in una situazione piena di luce, davvero 'solare'. Sempre al magistero dantesco sarà poi da ricondurre la scelta della terza rima, mentre non mancano vere e proprie allusioni intertestuali alla Commedia. Ai vv. 19-21 del secondo "capitulo" leggiamo:

Vento dal sonno, uno uomo de senno accorto me si fe innanti agli occhi e me dicea:

"A che pur gridi sì dal pianto scorto?" (Colelli-Marinelli 2018: 31)

187

Fabrica Litterarum Polono-Italica | 2020, nr 1 (2) 
È qui evidentissima la reminiscenza dantesca di Inf. V 21 "Perché pur gride?" e a questo punto, aggiungo a quanto giustamente osservato da Colelli, sarà del tutto casuale che tale reminiscenza cada proprio al verso 21?

Ancora: le navi del corteo navigano verso Fiume (III 108-111) e il poeta scrive:

Passaimo un golfo pria che il corso dome,

tal che lasciando da man destra Segne,

da l'altra Ossera e Cherse il gran paese, il porto presso Veglia ce ricegne.

(Colelli-Marinelli 2018: 37)

Questi versi potrebbero effettivamente, come suggerisce il curatore del testo, alludere a Inf. XXVI 110-111: "da la man destra mi lasciai Sibilia, / dall'altra già m'avea lasciata setta" (Alighieri 2013: 419).

Fin qui qualche dantismo, ma non mancano nemmeno reminiscenze ariostesche, come nel caso di XI 40-41 ("Qui non potria nisciun con prosa o rima / narrar I'umanità di quella diva"), da confrontare con $O F \mid 2$, 2: "Cosa non detta in prosa mai né in rima" (Ariosto 2001: 1).

Per parte mia vorrei segnalare come di fronte al primo "capitulo", che ai versi 73-75 descrive la partenza di Bona Sforza con movenze da planctus epicediale ("Hor piangan li poeti, e con ogni arte / empian de stridi et angoscioso pianto / le bianche loro e celebrande carte", Colelli-Marinelli 2018: 28) di derivazione classica (cfr. ad es. Cat. III 1: Lugete, o Veneres Cupidinesque; Ov., Am. II 6, 3: Ite, piae volucres, et plangite pectora pinnis), poi riprese anche dalla tradizione medievale (Petrarca in morte di Cino da Pistoia, RVF XCII 1-2: "Piangete, donne, e con voi pianga Amore, / piangete, amanti, per ciascun paese", Petrarca 1996: 448), l'impressione sia quella di avere a che fare con un rovesciamento dell'epitalamio, con toni simili a quelli di loannes Secundus (Jean Second), El. I 8. Giulia, la donna amata dall'io lirico, è andata in sposa al rivale e allora, in un'elegia che è, appunto, un epitalamio rovesciato, Secundus si augura che, esattamente come nel caso della partenza di Bona, le divinità siano contrarie a quanto sta succedendo, scatenando temporali e tempeste. Scrive il poeta (11-20):

Phoebe, coloratum, si te bene credimus olim

Esse sacris patrem vatibus, abde caput.

At tu tela manu iaculare corusca rubenti

luppiter: hac sola luce sit alba dies.

Tuque lovis coniunx, quantum connubia cures

Talia, demissis amnibus ipsa doce.

Fallimur? An certe condit sua Cynthius ora?

Condit, et obscura nocte teguntur agri,

188

Fabrica Litterarum Polono-Italica | 2020, nr 1 (2) 
Praecipitantur aquae, mare pendet in aere densum, Ignibus excussis dissiluere poli. (Second 2005: 164)

L'elegia di Secundus data al 1531 (Second 2005: 166), quindi è antecedente alla pubblicazione del poemetto di Carmignano, ciò che però non autorizza a postulare una relazione diretta tra i due testi, tanto più che paiono mancare punti di contatto testuali cogenti, eccetto forse nel caso dell'esordio del poema ("L'ora che Phebo a noi suo volto asconde"), da confrontare con Phoebe [...] abde caput di Secundus. Vorrei limitarmi a offrire uno spunto per eventuali future ricerche indirizzate a sondare la dimensione intertestuale dell'operetta, che potrebbe riservare sorprese.

Non solo dunque un poemetto odeporico, ma anche un epitalamio, che inizia come epitalamio negato per poi concludersi con le nozze.

Chiudono il volume alcuni sonetti del Carmignano, che il curatore ha meritoriamente offerto al lettore moderno. Si tratta di sonetti tipicamente petrarcheschi, di schema ABBA ABBA CDC DCD. Questi testi, se pur non sempre pienamente riusciti dal punto di vista artistico, hanno una fondamentale importanza storica, giacché potrebbero probabilmente aver giocato un ruolo non indifferente nell'introdurre in Polonia la forma sonetto, che ad ogni modo si diffonderà, com'è noto, soltanto a partire dalla fine del XVI secolo (Pszczołowska 1993). A conclusione delle mie osservazioni su questa meritoria e ben riuscita impresa editoriale, riporto le parole che Jan Ślaski impiegò a descrivere il seguito di Bona Sforza e l'influenza che questa ebbe sulla vita culturale polacca (Ślaski 1980: 342):

Fra i nuovi venuti, prevalevano però gli scrittori di minor levatura, oggi in genere dimenticati [...]. Ma non bisogna trascurare neppure questi autori prevalentemente di circostanza, in quanto anche le loro opere contribuivano a formare il quadro della cultura letteraria aulica. E tale cultura doveva essere assai estesa, se perfino i medici si occupavano di letteratura, ottenendo risultati non fra i peggiori.

\section{Bibliografia}

AlighieRI Dante (2013): Inferno. A cura di S. Bellomo. Torino.

Ariosto Ludovico (2001): Orlando Furioso. A cura di C. Segre. Milano.

PszczoŁowsKa Lucylla (1993): Sonet od renesansu do Młodej Polski. W: Słowiańska metryka porównawcza. T. 5. Red. L. Pszczołowska, D. Urbańska. Warszawa.

Nuovo Isabella (2007): Viaggio de la Serenissima S. Bona Regina da la sua arrivata in Manfredonia andando verso del suo regno di Polonia / Parthenopeo Suavio

189

Fabrica Litterarum Polono-Italica | 2020, nr 1 (2) 
(Colantonio Carmignano). A cura di Isabella Nuovo. Edizioni digitali del CISVA parzialmente riprodotto [ultimo accesso: 23.04.2020].

Petrarca Francesco (1996): Canzoniere. Edizione commentata a cura di Marco Santagata. Milano.

ŚLASKI Jan (1980): I poeti neolatini del primo umanesimo in Polonia e l'Italia (qualche proposta di ricerca). In: Italia, Venezia e Polonia tra Medio Evo e età moderna. A cura di V. Branca, S. Graciotti. Firenze.

SECOND Jean (2005): Oeuvres complètes. T. 2: Elegiarum Libri Tres. Edition critique établie et annotée par Roland Guillot. Paris.

\section{Abstrakt \\ Kilka refleksji na temat nowego wydania Viaggio della Serenissima S. Bona Regina in Polonia C. Carmignana}

Artykuł odsłania genezę i budowę tekstu Colantonia Carmignana. Przedstawia też ciekawą przedmowę, którą do książki napisał Luigi Marinelli. Proponowane wydanie zawiera także propozycje co do intertekstualnego wymiaru wiersza, zwracając uwagę na podobieństwa między pierwszym wersem utworu a elegią I 8 Joannesa Secundusa, przy czym oba teksty zostały pozbawione cech epitalamium.

Słowa kluczowe: Carmignano, Bona Sforza, Andrea Colelli, Luigi Marinelli, literatura polska, literatura włoska 